\title{
Effects of compressibility and shock-wave interactions on turbulent shear flows
}

\author{
Neil D. Sandham \\ Aerodynamics and Flight Mechanics Group \\ Faculty of Engineering and the Environment, University of Southampton \\ Southampton SO17 1BJ, UK \\ n.sandham@soton.ac.uk
}

April 13, 2016

\begin{abstract}
Compressibility effects are present in many practical turbulent flows, ranging from shockwave/boundary-layer interactions on the wings of aircraft operating in the transonic flight regime to supersonic and hypersonic engine intake flows. Besides shock wave interactions, compressible flows have additional dilatational effects and, due to the finite sound speed, pressure fluctuations are localized and modified relative to incompressible turbulent flows. Such changes can be highly significant, for example the growth rates of mixing layers and turbulent spots are reduced by factors of more than three at high Mach number. The present contribution contains a combination of review and original material. We first review some of the basic effects of compressibility on canonical turbulent flows and attempt to rationalise the differing effects of Mach number in different flows using a flow instability concept. We then turn our attention to shock-wave/boundary-layer interactions, reviewing recent progress for cases where strong interactions lead to separated flow zones and where a simplified spanwise-homogeneous problem is amenable to numerical simulation. This has led to improved understanding, in particular of the origin of low-frequency behaviour of the shock wave and shown how this is coupled to the separation bubble. Finally, we consider a class of problems including side walls that is becoming amenable to simulation. Direct effects of shock waves, due to their penetration into the outer part of the boundary layer, are observed, as well as indirect effects due to the high convective Mach number of the shock-induced separation zone. It is noted in particular how shock-induced turning of the detached shear layer results in strong localized
\end{abstract}


damping of turbulence kinetic energy.

\section{Introduction}

While shock-wave interactions with turbulent boundary layers were known since basic experiments conducted in the 1940s (Dolling, 2001), it became apparent in subsequent work that more subtle effects of compressibility were also present, for example in the experimental data on mixing layers collated by Birch \& Eggers (1973) that showed a large reduction in growth rate as the Mach number increased. Indeed this was one of the motivations for the Brown \& Roshko (1974) work on mixing layers that, besides stimulating work on organised structures in turbulence, also demonstrated that the reductions in growth rate could not be attributed solely to the effects of variable density. However other flows, such as attached turbulent boundary layers, did not show strong effects of compressibility, with Morkovin's hypothesis (Bradshaw, 1977) of weak compressibility applying up to Mach 5. Some of the apparent contradictions have been resolved in subsequent research, while some aspects, including turbulence and sub-grid modelling for compressible flows, are unresolved. A number of reviews of compressible turbulence and shock-wave/boundary-layer interaction (SWBLI) have been published over the last decade, including Smits \& Dussauge (2006), Gatski \& Bonnet (2009), Babinsky \& Harvey (2011) and Clemens \& Narayanaswamy (2014). Recent progress on understanding low-frequency unsteadiness of SWBLI interactions, along with heat transfer and flow control, has been reviewed by Gaitonde (2015).

In this contribution we will first consider (in section 2) the main canonical flow problems and then discuss flow stability arguments that, in connection to turbulence production at the largest eddy scales, can help to explain some of the differences seen between different flows such as the mixing layer and wall boundary layer. We then review (in section 3) more practical examples of SWBLI including fully 3D flow where the need to incorporate additional physics is observed in the form of streamline curvature effects.

Much of the recent progress has come from improvements in both experimental flow diagnostics and in the capabilities of numerical simulations based on eddy-resolving techniques such as direct numerical simulation (DNS) and large-eddy simulation (LES). Over the last 25 years experiments have been revolutionised by the application of particle image velocimetry (PIV) and related techniques such as stereo PIV. Initially developed for low-speed flows, the techniques are now being applied to high-speed SWBLI. Examples of the emerging capabilities of experiments are seen in recent studies of SWBLI by Souverein et al. (2010) for turbulent 
interactions and Giepman et al. (2015) for initially laminar interactions. On the numerical side progress has been even more striking, mainly enabled by improvements in computer hardware, but also with careful attention to algorithms, so this is briefly reviewed first, before proceeding to the physical problems.

\subsection{Progress in numerical simulations of SWBLI}

In this section we focus only on the techniques of DNS and LES that can provide reliable access to time-dependent three-dimensional flow fields during SWBLI. A pioneering study was by Adams (1998), who considered ramp flow at Mach 3, including a fully turbulent inflow based on a precursor simulation of a temporally-developing boundary layer. The numerical approach used compact finite differences (6th order) and an essentially non-oscillatory (ENO) scheme for shock capturing. Adams (2000) used the methodology to resolve a small separation bubble in the ramp corner, the high-frequency components of shock oscillation and the strong amplification of turbulence energy in the interaction region.

A fundamental numerical issue in SWBLI is the requirement both to capture shock waves and resolve turbulence to an acceptable level. Shock-capturing schemes are dissipative by nature and, as a by-product, damp turbulence such that acceptable accuracy may only be achieved with extremely fine grids. A pragmatic approach is usually adopted, as suggested for example by the work of Yee et al. (1999), which showed the benefits of additional shock detectors that serve to restrict the additional dissipation to the immediate vicinity of the shock waves. Even with such localisation, no benefit was seen in moving from fourth to higher order of accuracy. Given the additional inter-processor communication costs of compact schemes, limiting the order of accuracy to fourth order and using non-compact schemes has led to efficient algorithms that scale well to large core counts on massively parallel architectures. Additional ad hoc limiters such as that of Ducros et al. (1999) serve to restrict the dissipative shock-capturing schemes within boundary layers. This is potentially an issue where shock waves penetrate boundary layers, an example of which will be seen later in this paper. An unwelcome feature of these additional limiters is the appearance of additional numerical parameters that one might be tempted to tune for each simulation. However, this is not advisable and a better approach is to fix all the parameters for model problems and then numerical issues, when they appear, can usually be attributed to a lack of grid resolution.

Other algorithmic developments over the last 20 years have led to more stable schemes for high Mach number, a number of which are compared by Johnsen et al. (2010). Examples of the 
algorithms used in SWBLI applications include the entropy splitting appraoch fromGerritsen \& Olsson (1996) that was applied to turbulence simulation in Sandham et al. (2002), and skew-symmetric formulations, reviewed by Pirozzoli (2011). The idea is to ensure that some energy-like norm of the flow solution is conserved, although typically this is only provable for simplified forms of the equations. Other stabilising features include treatment of odd-even decoupling, which would otherwise lead to grid-to-grid point oscillations and explicit filtering that is often incorporated in place of the de-aliasing approach that would commonly be applied in spectral simulations of incompressible flows. Again, caution is needed, since filtering can lead to smooth solutions, giving the impression that a flow is fully resolved. It may be better in many applications to have a numerical method that leads to grid-scale oscillations when the grid is inadequate, since then the problem is apparent and in principle fixable with a finer grid, even though this comes with additional computational cost. Recent works commonly use shock capturing from one of the family of weighted ENO (WENO) schemes, but are computationally expensive (Pirozzoli, 2011) and Johnsen et al. (2010) caution that grid refinement studies are unavoidable for such schemes.

Large-eddy simulation is problematic for compressible flows due to the large number of sub-grid terms that may need to be modelled, as discussed in Garnier et al. (2009). Vreman (1995) recognised the problem and overcame it to some extent by proposing dynamic models that do not require any particular physical insight into the nature of the various terms. On the other hand, the errors due to the neglect of compressibility effects may not so serious for LES, since most of the strong compressibility is expected to reside in the larger scales that are resolved on the grid. The majority of examples of LES for high-speed flows use sub-grid models that have been converted from low speed flows in a straightforward manner by including density variations. Sub-grid heat conduction is usually treated with a constant turbulent Prandtl number. A big variable in LES is the size of the grid relative to what would be required for a DNS. For flows near wall a common approach is to resolve the flow down to the structures of the viscous sublayer, which means that grids are typically within a factor of 2 to 4 (in each direction) of the grids required for DNS. In this situation relatively little energy is carried in the subgrid model and errors to the omission of compressibility terms will anyway be small. Nevertheless, shocks and eddy shocklets have spectral content at the smallest scales and this is not generally taken into account in the models.

A recent research direction in numerical approaches to turbulence and shock waves has been to avoid sub-grid models entirely by adopting numerical dissipation from the basic numerical algorithm. At its crudest this approach involves turning off the subgrid modelling and 
using the shock-capturing method as a sub-grid treatment, essentially providing sufficient dissipation to avoid a pile-up of energy in the small scales of turbulence. More elegant approaches involve adapting the characteristics of the numerical scheme to represent the sub-grid turbulence, a recent example of which is given by Hickel et al. (2014), where a compression ramp flow was computed using a numerical method that simultaneously captured shock waves and was consistent with known properties of incompressible turbulence. In a comparison with a dynamic Smagorinsky calculation for a shock-turbulence interaction problem, equivalently good results were obtained. An additional aesthetic advantage of such methods is the lack of tunable parameters in the algorithm.

\section{Canonical Flows}

In this section we discuss some results for two simple flows that exhibit strong compressibility effects, namely mixing layers and turbulent spots, and contrast this behaviour with zero pressure gradient boundary layers which show minimal compressibility (as opposed to mean density) effects up to free stream Mach numbers of around 5. In the final subsection we rationalise this behaviour by considering flow instabilities and extrapolate the results to boundary layers with adverse pressure gradients, where true compressibility effect are expected for much lower free stream Mach numbers.

\subsection{Mixing layers}

The most striking effect of compressibility on turbulence is the reduction in growth rate of the compressible turbulent mixing layer. Experiments by Papamoschou \& Roshko (1988) showed how a convective Mach number concept, together with a suitable normalisation of mixing layer growth rate with its incompressible value, could collapse data over a wide range of Mach numbers and density ratios. For free-streams with equal ratio of specific heats, the convective Mach number is given by $M_{c}=\left(U_{1}-U_{2}\right) /\left(a_{1}+a_{2}\right)$, where $U$ is the velocity and $a$ the sound speed, with subscripts 1 and 2 denoting the fast and slow speed streams respectively. $M_{c}$ can be interpreted as the free-stream Mach number seen by an eddy convecting downstream at a velocity of $U_{c}=\left(a_{2} U_{1}+a_{1} U_{2}\right) /\left(a_{1}+a_{2}\right)$. The most comprehensive attempt to collapse the experimental data is in Slessor et al. (1998), who used a modified version of the convective Mach number to get a best fit. For two parallel streams with equal ratio of specific heats and equal free stream temperatures the resulting curve is shown in figure 1, in the form of a plot of the relative growth rate $\Phi$ (the mixing layer spreading rate under compressible flow conditions 


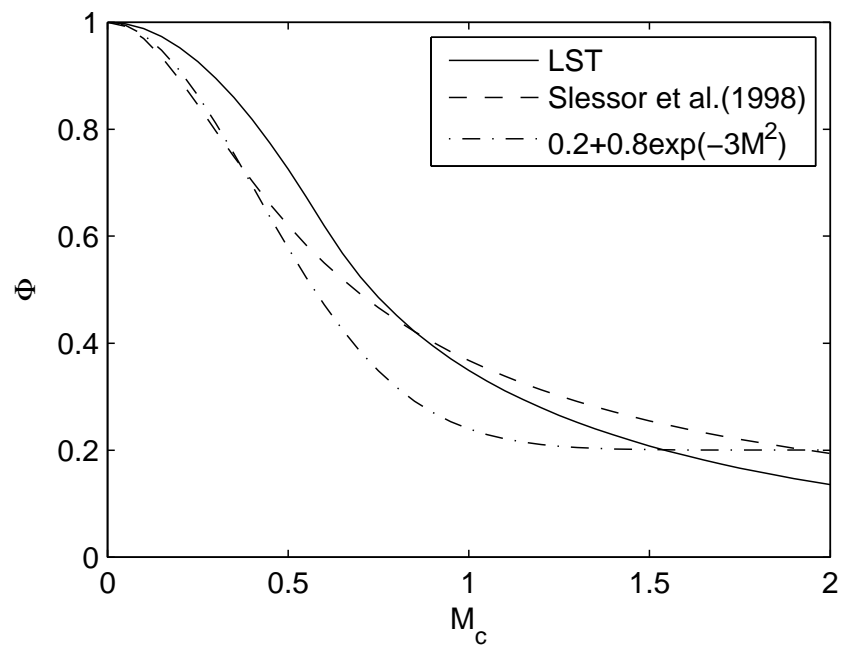

Figure 1: Relative growth rate $\Phi$ as a function of convective Mach number $M_{c}$, showing results from linear stability theory (LST) compared with the empirical fits to experimental data discussed in Slessor et al. (1998).

divided by the spreading rate in incompressible flow for the same velocity and density ratio) against the convective Mach number $M_{c}$. The growth rate of the turbulent wedge is seen to be reduced by almost a factor of three as the convective Mach number is raised to 1.0. Despite a considerable spread in the experimental data, the convective Mach number concept appears to be well supported by experimental data. Figure 1 also shows a curve from linear stability theory (LST), showing the effect of Mach number on the temporal growth rate (taking the maximum over all streamwise and spanwise wavenumber combinations). We shall return later to the observation that the linear theory follows the empirical fits quite closely, at least considering the scatter of the experimental data.

An explanation for the reduction in growth rate has been sought from the Reynolds stress transport equations. Early suspicions fell on the additional terms in these equations that are not present in incompressible flows. However, Vreman et al. (1996) showed that the dilatation dissipation and pressure dilatation terms remained small even as the growth rate reduced significantly due to compressibility. It was shown instead how the growth rate falls in proportion to the production and that this in turn was caused by reductions in pressure strain terms in the Reynolds stress equations. Thereafter, explanations differ as to the root cause. Vreman et al. (1996) considered a model compressible vortex, showing how pressure fluctuations naturally reduced in compressible flows. A model based on this idea, together with the concept of sonic-limited eddies originally proposed by Breidenthal (1992), was shown to capture the growth rate reduction. A different perspective was offered by Pantano \& Sarkar 
(2002), who considered the effect of reduced communication due to the finite sound speed.

The state of turbulence modelling for compressible flows is discussed in Wilcox (2006), which despite being ten years old still represents the state of the art. Wilcox describes a number of fixes to low-speed models to account for both the rapid change of mixing layer growth rate with Mach number and the relative insensitivity of the boundary layer to Mach number. These fixes are purely empirical and the lack of progress in modelling has been a disappointment. One approach was based on dilatation dissipation, for example in Sarkar et al. (1991), which considered the additional dissipation due to eddy shocklets. Some ad hoc attempts have since been made to damp pressure strain terms with Mach number, as reviewed by Yoder et al. (2015). However, the modelling of compressibility based on the physical understanding gained from DNS has not so far been translated into practical models the can be implemented in commercial Computational Fluid Dynamics (CFD) codes and, as such, results from such codes must be treated with extreme caution. With further advances in experimental diagnostics and DNS it is now possible (Barre \& Bonnet, 2015) to develop reliable kinetic energy budgets in spatially-developing mixing layers at $M_{c}=1$, providing for the first time the complete databases needed to reappraise the modelling issues.

\subsection{Boundary layers}

It is well known that compressible wall boundary layers are mainly affected by density effects, rather than compressibility, for free stream Mach numbers $M_{\infty} \leq 5$ (Bradshaw, 1977). The standard way to scale density effects out of the mean profile is with the van Driest transformation, given by

$$
u_{\mathrm{vD}}^{+}=\int_{0}^{u^{+}}\left(\frac{\rho}{\rho_{w}}\right)^{1 / 2} d u^{+}
$$

The transformed profiles have been observed to provide a useful (though not exact) collapse of high speed boundary layer profiles back onto incompressible cases, as shown for example in Duan \& Martin (2011). The transform has also been used to extend turbulence inflow generation methods to fully compressible flow, for example in Touber \& Sandham (2009).

Early progress on understanding the effect of compressibility on boundary layer turbulence was reviewed by Bradshaw (1977). He expressed the findings as a number of hypotheses and analogies between energy and momentum transport. Morkovin's hypothesis covers an expectation that the turbulence shear stress and streamwise normal stress are not strongly dependent on Mach number, whereas the strong Reynolds analogy uses a linearisation of the governing equation to connect thermodynamic and velocity fluctuations (see Gatski \& Bonnet 
(2009) for a complete discussion). Evidence for the applicability or otherwise of these relations is provided by DNS studies, including Maeder et al. (2001) and Pirozzoli et al. (2004). In particular, Huang et al. (1995) and Duan \& Martin (2011) show how the analogies can be adapted for high Mach number and high enthalpy flows to provide reasonable representations of the turbulence in attached wall flows.

Other insights have helped to understand the different effects of Mach number on different flows. In particular, the gradient Mach number $M_{g}=S l / a$ (where $S$ is the mean shear rate, $l$ is an integral lengthscale of the turbulence and $a$ is the sound speed) was exploited by Sarkar (1995) to compare mixing layers with boundary layers, since it was found that for equivalent flow Mach numbers the gradient Mach number was substantially higher in mixing layers. This study also first identified the role of compressibility in modifying the production rate of turbulence.

\subsection{Turbulent spots}

Given the relative insensitivity of zero pressure gradient boundary layers to the free-stream Mach number, it is surprising that the lateral growth rate of turbulent spots (regions of a turbulence within an otherwise laminar boundary layer) is strongly affected by compressibility. Figure 2(a) shows an example of a turbulent spot simulated at Mach 3 with an adiabatic wall boundary condition (Redford et al., 2012). The spot spreads approximately linearly as it moves downstream, with a half angle defined relative to an artifical origin upstream, which needn't be the spot inception point. A collection of experimental data by Fischer (1972) shows a reduction in spreading angle by a factor of three at $M_{\infty}=5$, compared with incompressible flow (where the spreading half angle is about $10^{\circ}$ ). In the years since the experimental data was published, it has become possible to carry out numerical simulations of some of the cases, with examples reported in Krishnan \& Sandham (2006) and Jocksch \& Kleiser (2008). It is interesting to note that there are varying definitions of spot width and numerical simulations show similar scatter to the experiments, as shown in figure 2(b). Calculations by Redford et al. (2012) show the expected reductions in growth rate and how the wall thermal condition is of lesser importance than the Mach number. Cases with cold walls (wall temperature equal to the free stream temperature) spread more slowly than spots in flows where the walls are at the adiabatic temperature, but only by $20-30 \%$, whereas the spreading rate at $M_{\infty}=6$ is already reduced by a factor of four compared to incompressible flow.

Why should compressibility be so important to the spreading of turbulent spots, but 


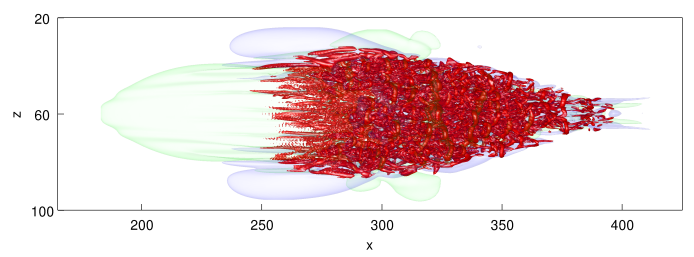

(a)

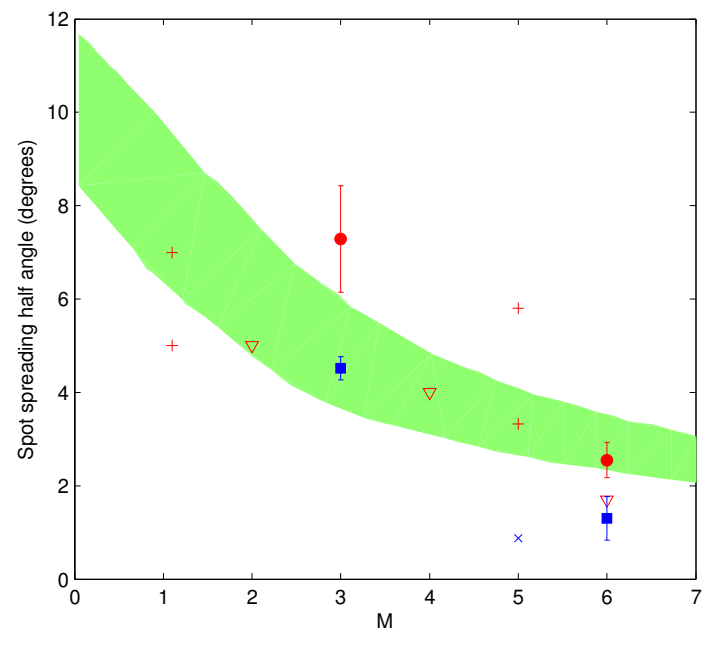

Figure 2: Structure and spreading rate of turbulent spots (Redford et al., 2012): (a) Turbulent spot at Mach 3 with an adiabatic wall condition. Red shows the second invariant, green the high streamwise momentum of the calmed region and blue the low streamwise momentum caused by lift-up at the spot wing tips. (b) Variation of the spreading angle of the half-width of turbulent spots as a function of free-stream Mach number. The shaded area represents the spread of the experimental data from Fischer (1972), while the symbols show DNS results from Jocksch \& Kleiser (2008) (+), Krishnan \& Sandham (2006) (open symbols) and Redford et al. (2012) (filled symbols, red for hot wall conditions and blue for cold-wall conditions).

play only a minor role in the fully developed turbulent boundary layer? To explain this, attention has focused on the wing-tip region where the boundary between turbulent and laminar flow provides a strong lateral velocity gradient that can drive the spot growth. GadEl-Hak et al. (1981) noted that the turbulence can spread by two mechanisms, either by mean convection outwards from the spot centre, or by destabilisation of the surrounding boundary layer. Once fluid has become turbulent it can be thought of as being convected at typical speeds associated with structures within the turbulent flow. Structures towards the edge of the boundary convect at near the free stream speed, while those closest to the wall (the near wall streaks and buffer layer structures) convect at speeds of 40-50\% of the free stream, dependent on Reynolds number. This leads to a natural streamwise spreading of the 
newly-turbulent flow that, together with the lateral spreading, gives the classical arrow-head structure of spots. Close to the wall at the rear of the spot the turbulent flow (with high skin friction) merges back into a laminar boundary layer (with lower skin friction). In this region there is a large positive $\partial u / \partial x$ and for mass conservation along the spot centreline there must be a negative $\partial v / \partial y$, leading to an inrush of high momentum fluid behind the spot. This produces a fuller velocity profile behind the turbulent region that is stable and known as the calmed region. More complicated explanations of the formation of the calmed region can be found in the literature, for example Brinkerhoff \& Yaras (2014), but the simple explanation given here shows the basic phenomenon. The turbulent structure is changed by compressibility, with examples of turbulent spots shown in Redford et al. (2012). Additional structures related to the second (Mack) modes of instability are also observed underneath the spots (Krishnan \& Sandham, 2006). After being first observed in DNS, these structures have now also been observed in laboratory experiments by Casper et al. (2014). With respect to the part of the growth mechanism that was affected by compressibility, Redford et al. (2012) showed how it was the destabilisation of the surrounding boundary layer (Gad-El-Hak's second mechanism) that was affected by Mach number, with a much slower rate of generation of new structures at Mach 6 compared to Mach 3 in their simulations. The relevance of an instability/destabilisation mechanism for spot growth is something that we will follow up at the end of the next subsection.

\subsection{Synthesis of canonical flow results in terms of a $\theta-M_{c}$ stability map}

There have been a number of studies that make a connection between local stability analysis and turbulent free shear layer growth rates, of which Monkewitz \& Huerre (1982) is an early example. In particular the spatial growth rate of small amplitude disturbances is found to be approximately proportional to the growth rate of a turbulent mixing layer. The rationale for this, that the growth and decay of long-wavelength structures (more than ten times the mixing layer vorticity thickness) controls the production of turbulence at large scales, was tested experimentally by Gaster et al. (1985), who showed that upstream forcing of instability waves could be quantitatively compared to the downstream behaviour of large structures in mixing layers. This was elaborated by Morris et al. (1990), who showed how the proportionality between linear spatial growth rate and mixing layer growth rate could be derived from a simplified model. Further evidence for the continued importance of linear modes has emerged in the study of jet noise; for example Suzuki \& Colonius (2006) measured disturbances in the potential flow just outside a circular jet, with clear detection of the linear eigenmodes of the 


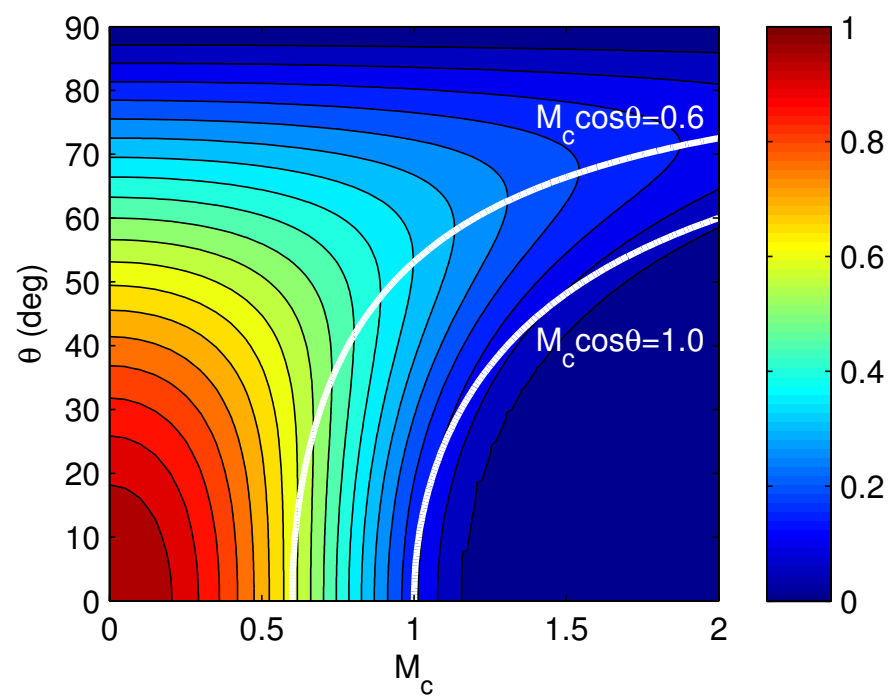

Figure 3: Contours of disturbance growth reduction factor $\Phi$ as a function of wave angle $\theta$ and convective Mach number $M_{c}$.

(turbulent) jet shear layer.

For compressible flows, the relation between mixing layer growth rate and spatial stability theory was noted by Ragab \& Wu (1989) and Sandham \& Reynolds (1990). In particular it was shown how the effect of velocity ratio, density ratio and Mach number on mixing layer growth rate could all be well predicted by the linear theory. In this section we present the basic shear layer instability results in the form of a $\theta-M_{c}$ map, where $\theta$ is the wave angle and $M_{c}$ is the convective Mach number, and we will attempt to find definitions of an effective $M_{c}$ (different to the gradient Mach number $M_{g}$ ) for boundary layers and turbulent spots.

The basic $\theta-M_{c}$ plot is shown on figure 3. The vertical axis is the wave angle $\theta$, which is equal to zero for spanwise wavefronts and $90^{\circ}$ for streamwise wavefronts. The horizontal axis is the convective Mach number $M_{c}$ defined earlier. The contours on the stability map are of the growth reduction factor $\Phi$, which is defined as the growth rate relative to the largest growth rate of the same case (with the same velocity and density ratio) from incompressible flow. $\Phi$ is defined by taking the maximum growth rate over all streamwise wavenumbers. In Sandham \& Reynolds (1990) it was shown that the spatial and temporal theories provide very similar results for $\Phi$, so here we use the temporal approach for simplicity and only consider cases with equal density ratio. The plot includes only first mode disturbances, which are oblique at the higher Mach numbers. There are also radiating modes, but these are only weakly unstable and can be ignored in this context. The first stages of oblique-mode breakdown to 
turbulence were presented based on DNS by Sandham \& Reynolds (1991), and it is interesting that such mode combinations are also relevant to boundary layer transition in the supersonic flow regime (Mayer et al., 2011), before additional second (Mack) modes enter the problem at higher Mach numbers.

A number of interesting results are clearly seen from figure 3 . At $M_{c}=0$ the most unstable mode is 2D (a consequence of Squires' theorem). This is not to say that only 2D modes are excited, however. An $N$-factor calculation in Sandham \& Sandberg (2009) showed how structures could emerge from background spectra including structures at different angles, not just for $\theta=0^{\circ}$. Neither this approach, nor the DNS, gave perfectly spanwise structures, implying that other factors may have been active in the Brown \& Roshko (1974) experiments that showed clear spanwise organisation. From figure 3 it can be seen that for $M_{c}>0.6$ oblique modes are the most unstable. The peak growth rate follows a line $M_{c} \cos \theta=0.6$ (Sandham \& Reynolds, 1990). The variation of $\Phi$, taking the maximum over $\theta$ for each $M_{c}$ was shown on figure 1 as the LST result. This curve matches the observed decrease in shear layer growth rate observed experimentally, almost as well as the empirical fit to the data. Finally we note that for $M_{c} \cos \theta>1$ (the second contour shown on figure 3 ) there is no first mode growth, supporting the sonic-eddy suggestion of Breidenthal (1992)) that only subsonic eddying motions are relevant. Thus, for any local shear layer that is evolving within a turbulent flow, a dead zone occurs, with no instability (up to a certain wave angle) to drive shear-layer roll-up. This is expected to modify the turbulence dynamics at any scale where a locally-defined convective Mach number exceeds one.

The stability plot extends straightforwardly to other free shear layers such as jets and wake. However, we will also attempt to show in the following paragraphs how it may potentially also be applied to boundary layers, particularly under the effect of adverse pressure gradients, and to turbulent spots, by a suitable redefinition of $M_{c}$. The potential for a more general applicability of these concepts is supported by the recent work of Kumar et al. (2014) who considered pressure-velocity interactions in homogeneous shear flows. They identified the key parameter as a Mach number $M_{g} \cos \theta$ and noted the stability of supersonic modes within the turbulent flow.

We have already remarked that compressibility effects enter the turbulence problem by modifying the production term, i.e. at the scale of the largest local structures, or alternatively where the local mach number fluctuations are largest. For the boundary layer, there are three regions where compressibility effects might enter, namely the buffer layer (which is exposed to a large velocity gradient), the log layer, and the outer (wake) region. Although 


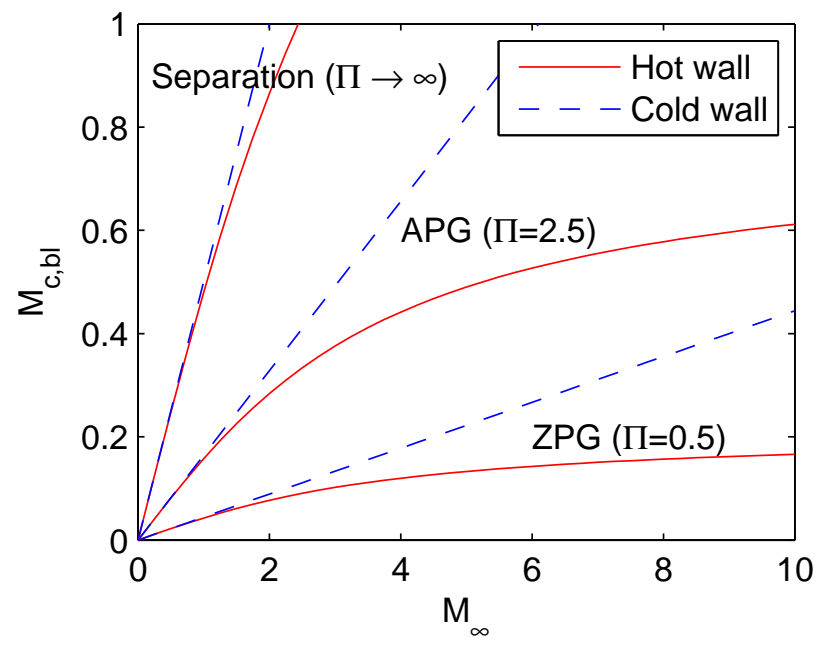

Figure 4: Effective Mach number $M_{c, \mathrm{bl}}$ according to equation (4), including the effects of adverse pressure gradient variation from zero to separation based on the wake parameter $\Pi$, and wall temperature ('hot' denotes an adiabatic wall and 'cold' denotes a case where the wall temperature is equal to the free stream temperature).

the buffer layer is in a region of high velocity gradients, it is also typically a region of high temperature and hence high sound speed. This is sufficient to reduce considerably the effective value of $M_{c}$, for example a Mach 5 boundary layer with a wall temperature equal to the adiabatic wall temperature (assuming a recovery factor of 0.9), a buffer layer $\Delta U^{+}=6$ (i.e. a very strong eddy) and an edge velocity of $U_{\infty}^{+}=25$ would have an effective Mach number of $M_{c}=0.05 M_{\infty}=0.25$, based on $M_{c}=\Delta U /\left(2 a_{w}\right)$ putting this within the region relatively unaffected by compressibility according to figure 3. Nevertheless, there can still be some effects of compressibility, particularly for cold-wall cases, such as the changes in the low speed streaks observed by Coleman et al. (1995). In the log layer we can make a simple estimation based on the idea of a mixing length $l=\kappa y$ (with $\kappa$ the von Karman constant). Here the effective Mach number, for a boundary layer with $U_{\infty}^{+}=25$ is given by $M_{\mathrm{c}}=M_{\infty} /\left(2 U_{\infty}^{+}\right) l^{+}\left(\mathrm{d} u^{+} / \mathrm{d} y^{+}\right)=0.02 M_{\infty}$, for the worst case of a constant density and hence constant sound speed equal to the free stream value $\left(a_{\infty}\right)$.

The outer layer is interesting because the form of the mean velocity profile that adds onto the log law to get to the observed data is already in the form of a mixing layer (i.e. Coles' wake function) as seen in the last term of the van Driest-transformed mean velocity equation, given by

$$
u_{\mathrm{vD}}^{+}=\frac{1}{\kappa} \ln y^{+}+b+\frac{\Pi}{\kappa}\left[1-\cos \left(\frac{\pi y}{\delta}\right)\right] .
$$


We can develop an effective Mach number using the wake parameter $\Pi$ and the respective sound speeds at the wall $\left(a_{w}\right)$ and in the freestream $\left(a_{\infty}\right)$ by

$$
M_{c, \mathrm{bl}} \equiv \frac{\Delta U_{v D}^{+}}{U_{\infty}^{+}} \frac{M_{\infty}}{\left(1+a_{w} / a_{\infty}\right)}
$$

where $\Delta U_{\mathrm{vD}}^{+}$refers to the last term in (2), taking the difference between $y=0$ and $y=\delta$ and $\delta$ is the boundary=layer thickness. Using the van Driest profile, this becomes

$$
M_{c, \mathrm{bl}}=\frac{2 \Pi / \kappa}{\left(2 \Pi / \kappa+(1 / \kappa) \ln \delta_{v D}^{+}+b\right)} \frac{M_{\infty}}{\left(1+a_{w} / a_{\infty}\right)} .
$$

Figure 4 shows a plot of this effective convective Mach number for boundary layers as a function of free stream Mach number $M_{\infty}$ for three values of $\Pi$ and two different wall thermal conditions. The effects of variations in the wake strength are illustrated for $\Pi=0.5$, corresponding to a zero pressure gradient $(\mathrm{ZPG})$ boundary layer, $\Pi=2.5$, corresponding to a strong adverse pressure gradient (APG), and $\Pi \rightarrow \infty$, corresponding to the point of separation. The two thermal conditions are a cold wall, with $T_{w}=T_{\infty}$ and an adiabatic (hot) wall with

$$
\frac{T_{w}}{T_{\infty}}=1+r \frac{(\gamma-1)}{2} M_{\infty}^{2}
$$

with the recovery factor $r=0.9$ and the ratio of specific heats $\gamma=1.4$. The calculations are shown for a value of $\left(1 / \kappa \ln \delta_{v D}^{+}+b\right)=25$. The horizontal line shows the level of 0.3 that we might take as the threshold above which compressibility effects may be expected to be significant. We see that this is reached for cold-wall conditions $\left(a_{w}=a_{\infty}\right)$ at $M_{\infty}=6$ for ZPG flow, reducing down to $M_{\infty}=1.8$ for the strong APG case and $M_{\infty}=0.6$ for a boundary layer on the point of separation or reattachment. The hot wall cases are similar except that the adiabatic wall case never seems to reach conditions where true compressibility corrections are needed, at least until Mach numbers where real gas effects need to be incorporated. Thus compressibility may be a significant effect in transonic flows involving separation. This is not particularly a new observation, but equation (4) seems to provide a rationale for future studies to look for strong compressibility effects in boundary-layer flows with adverse pressure gradients at moderate supersonic $M_{\infty}$.

Finally in this section we consider the growth of turbulent spots and the surprising result that even in zero pressure gradient boundary layers the lateral growth rate is strongly compressible, showing a reduction by a factor of four in a boundary-layer at $M_{\infty}=6$. The reasons for this strong reduction have not yet been fully explored. Redford et al. (2012) showed how compressibility mainly acted on the mechanism of destabilisation of the surrounding laminar 


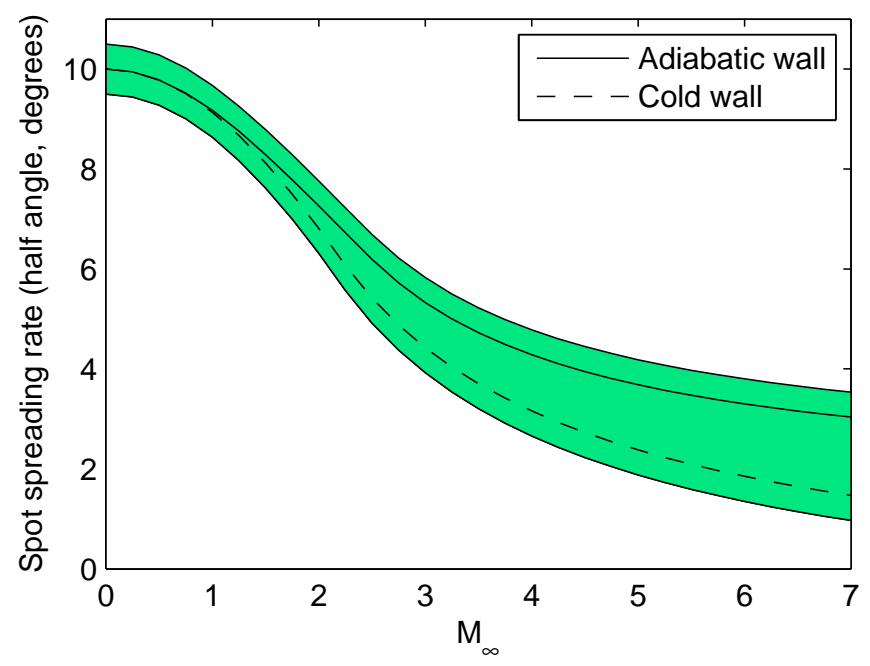

Figure 5: Prediction based on equation (6) of the growth rate (measured by the half angle in degrees) as a function of free stream Mach number $M_{\infty}$. Two cases are shown, for adiabatic and cold wall (wall temperature equal to free stream temperature) conditions. The green shaded zone correspond to an additional $5 \%$ tolerance for comparison with figure 2(b).

boundary layer and, perhaps most relevant to the mechanisms considered here, observed rollup of lateral jets emanating from the wing tips of the spot. The local instability problem is not amenable to analysis since the basic flow is three dimensional. Also, the jets themselves are not fast enough to be subject to high compressibility effects. However, a simple estimate of $M_{c}$ for these jets relative to the freestream can be derived, by considering the jet location in the laminar boundary layer, as

$$
M_{c, \text { spot }}=M_{\infty} \frac{1-U_{j} / U_{\infty}}{1+a_{j} / a_{\infty}},
$$

where $U_{j}$ is the laminar boundary layer streamwise velocity (and $a_{j}$ the corresponding sound speed) at the jet location and from Redford et al. (2012) $U_{j} \approx 0.45 U_{\infty}$. A prediction of spot growth rates based on equation (6) is shown on figure 5, where we take $U_{j}=0.45 U_{\infty}$ and consider two thermal conditions corresponding, as before, to hot (adiabatic) and cold (free stream) wall temperatures. The green shaded region is taken as the area between a line $5 \%$ above the hot-wall case and a line $5 \%$ below the cold wall case, and may be compared with the spread of experimental and numerical data from figure 2(b). The trends with both Mach number and wall temperature both seem to be captured by this method.

In this section we have attempted to extend the basic shear layer instability arguments from mixing layers to boundary layers with pressure gradients and to turbulent spots. These 
arguments are testable with more experiments and simulations of compressible boundary layers, particularly under conditions of adverse pressure gradient, and turbulent spots, where more understanding of the physical mechanisms of spot growth is needed, even in the more restricted case of incompressible flow, possibly along the lines of recent studies by Brinkerhoff \& Yaras (2014) and Goldstein et al. (2015).

\section{Shock-wave interactions}

\subsection{Shock/turbulence interaction without walls}

A useful starting point in considering the effect of shock waves on turbulence is a model problem consisting of isotropic turbulence passing through a normal shock, for which direct numerical simulations (DNS) and a linearised theory are possible. Linearised interaction analysis (LIA) is derived from the Euler equations. The oncoming turbulence can be decomposed into vortical, acoustic and entropy modes, which can in principle interact with each other according to the mechanisms given in Chu \& Kovásznay (1958). To study shock/turbulence interactions, each of the linear modes can be interacted separately with a normal shock wave. Results from comparisons between DNS and linear theory are reported in e.g. Mahesh et al. (1997), who looked in particular at the variations in turbulence associated with an entropy wave passing through a normal shock. Turbulence kinetic energy and temperature fluctuations are amplified across the shock, with the streamwise normal stress being most affected. Generally a good qualitative agreement has been found between DNS and theory and the DNS has been shown to converge to the LIA predictions just downstream of the shock in the limit of small turbulent Mach number (Ryu \& Livescu, 2014). However, Larsson et al. (2013) identified some important differences. The post-shock anisotropy of vorticity was found to be of small practical importance due to a rapid return to isotropy at higher Reynolds numbers. Additionally, different flow regimes in which the shock wave became wrinkled and broken were

identified. To help separate different physical phenonomena, it is useful to bear these results in mind when considering more complex interactions.

\subsection{Shock-wave/boundary-layer interactions}

When a shock wave interacts with a boundary layer the subsequent interaction can be weak, where the boundary layer is thickened by the adverse pressure gradient imposed by the shock, or strong, where there is a shock-induced boundary-layer separation. This category of canoni- 
(a)

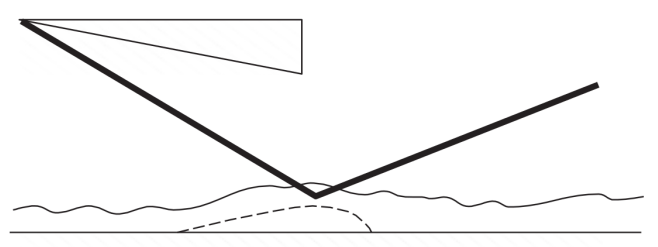

(b)

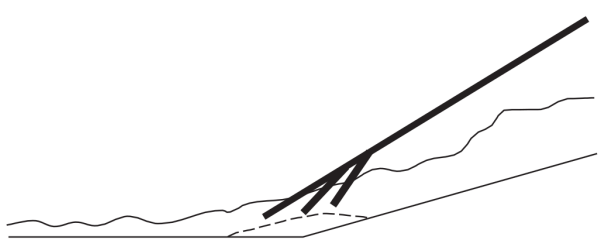

(c)

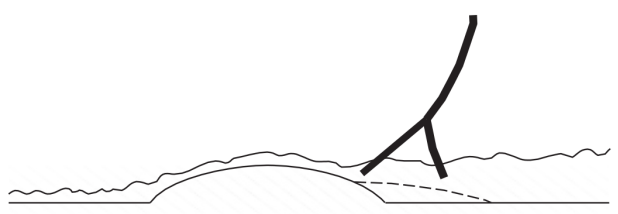

Figure 6: Three different types of shock-wave/boundary later interaction problems: (a) shock impingement (omiting details of the shock and expansion wave structure, (b) supersonic ramp flow, (c) transonic flow over a bump (Lawal, 2002).

cal flows can be further subdivided, as shown in figure 6, into: (a) an oblique shock impinging onto a boundary layer on a flat plate, (b) an interaction initiated by a ramp, and (c) a transonic interaction in flow over a bump. The latter case (simulated by Pirozzoli et al. (2010), for a case with incipient separation) is different, since the flow upstream and downstream is subsonic. However all three cases share important flow physics, including separation, with a shock wave developing from the coalescence of compression waves from the separation region, and a similar phenomenon leading to a reattachment shock, with a closed separation bubble forming in each case. The bump flow forms a classical $\lambda$-shock pattern, where the two shocks near the wall (forming the front and rear feet) meet at a triple point. Above this region a near-normal shock marks the end of the supersonic flow region. The oblique shock and ramp cases have similar physics, as shown for small disturbance cases by Pagella et al. (2004). However there are important potential differences, with many observations of streamwise structures attributed to Görtler instability due to the destabilising curvature near the reattachment point. There are also curvature effects in the oblique shock case, as we shall 
see later, and it is important to note that the flow over the top of the bubble in this case also experiences an expansion fan. The bubble in this case is roughly triangular in shape, with the apex at the point where the shock impinges and reflects as an expansion wave. For the oblique shock case, Matheis \& Hickel (2015) have considered stronger interaction cases where the regular reflection transitions to a Mach reflection.

All three cases in figure 6 show similarities in the difficulty of the turbulence modelling and in the occurrence of large amplitude low-frequency oscillations. The former problem is well known. Reynolds-averaged Navier-Stokes (RANS) methods give results of only qualitative accuracy, as for example shown by Doerffer et al. (2010). One might think that the unsteadiness can be captured in unsteady RANS (URANS), but this method only gives steady results, as does detached eddy simulation (DES). Better agreement with experiment can only be obtained with such methods by introducing additional unsteadiness, as shown by Garnier (2009), but this requires the specification of disturbances, the precise form of which is not known a priori. This is an ongoing research topic, as is the embedding of higher fidelity methods within RANS, URANS and DES. The lowest form of modelling that has been proven to work for SWBLI problems is LES, and strictly only with wall-resolved LES, by which we mean LES that resolves the sublayer, with total grid counts smaller than DNS by only a factor of 20-30 and with care taken particularly to resolve the wall-normal direction close to what would be needed for DNS. The first application of LES to the oblique SWBLI problem was by Garnier et al. (2002). Such simulations can show excellent agreement with experiment, as for example shown in figure 7, where results from Touber \& Sandham (2009) are compared with experiments carried out in Marseille by Dupont et al. (2006) and Piponniau et al. (2009). Figure 7(a) shows the mean streamwise velocity, with filled contours referring to experimental results and lines to simulations, while figure $7(\mathrm{~b})$ shows comparisons at different streamwise locations downstream of reattachment. We should bear in mind the sensitivity of the experimental flow to upstream conditions and sidewall effects, and of the LES to the spanwise domain size. Nevertheless, it is clear that LES captures the correct bubble physics. Furthermore, LES (as opposed to DNS) is the only currently feasible way of obtaining sufficiently long time series to be able to study the other interesting feature of shock-induced separated flow, namely the low-frequency unsteadiness.

There are a number of competing explanations of the occurrence of low-frequency oscillations in SWBLI problems, and it is only very recently that a resolution appears to be possible. Figure 8 shows a weighted (pre-multiplied with frequency) power spectrum of wall pressure fluctuations under the base of the reflected shock. The figure shows a good agreement be- 


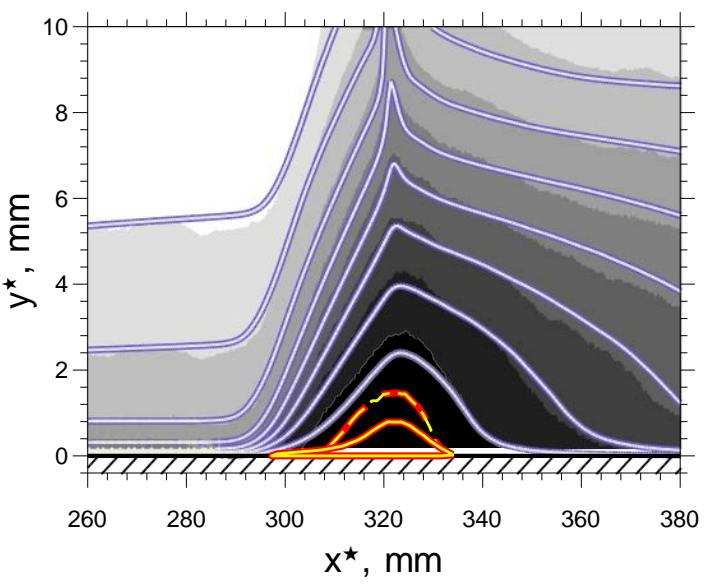

(a)

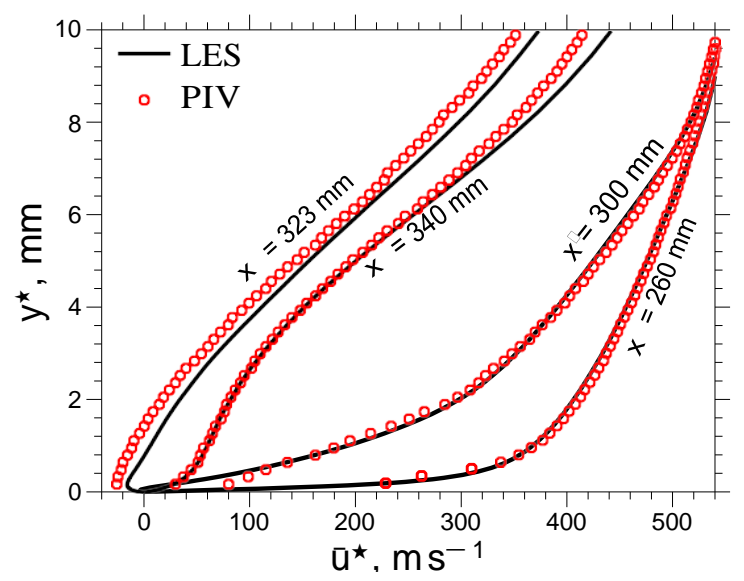

Figure 7: Comparison of LES with experiment showing (a) contours of mean streamwise velocity, comparing PIV (shaded) with LES (contour lines), with the orange lines (dashed for the experiment) showing the zero velocity contour, and (b) velocity profiles at several streamwise locations (Touber, 2010).

tween the experimental measurements (Dupont et al., 2006) and the LES. The LES shows two peaks, one at Strouhal number of $S t=0.03$ and the other (not resolved in the experiment) corresponding to boundary layer turbulence. Alternative explanations for the low-frequency peak include upstream boundary-layer disturbances, global modes, acoustic feedback mechanisms within the bubble and bubble 'breathing'. We will not recap all the arguments here; they are readily available in the literature, for example in Ganapathisubramani et al. (2007), Robinet (2007), Pirozzoli \& Grasso (2006), Piponniau et al. (2009) and recently reviewed by Gaitonde (2015). Each study is in some sense correct in that the physical phenomena are reproducible, but there is generally a lack of testable predictions from the various approaches. A resolution appears possible when we consider some recent developments. Firstly Touber \& 


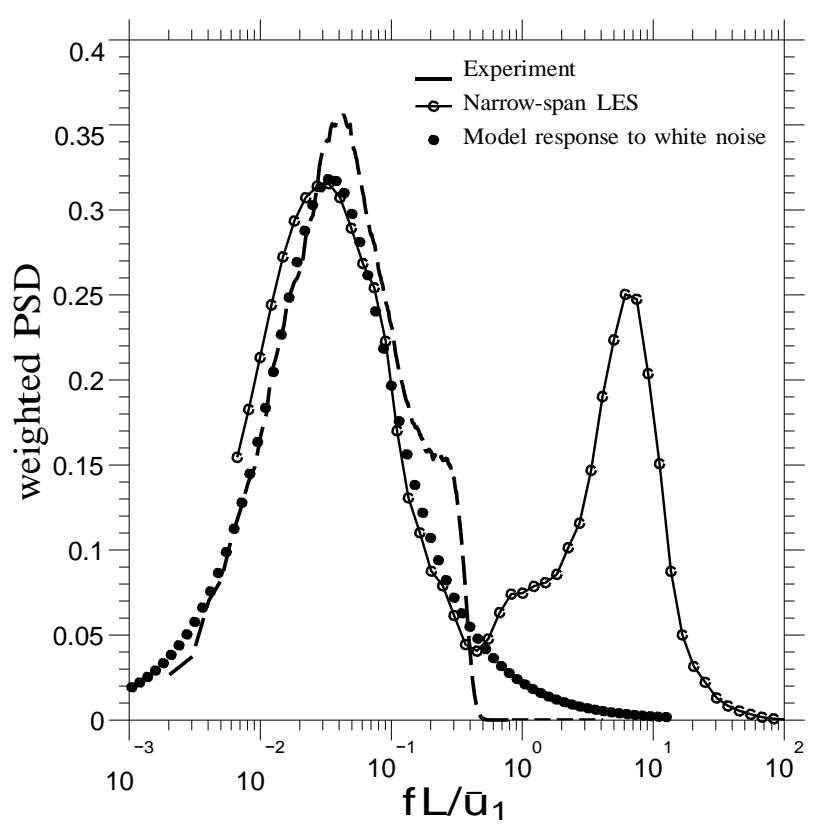

Figure 8: Weighted power spectral density of wall pressure fluctuations as a function of normalised frequency, comparing experiment (dashed line), LES (line with open symbols and the model (filled symbols), from Touber (2010).

Sandham (2011) showed how an analysis based on the (wall-normal) integrated Navier-Stokes equations, using LES data to neglect terms with lower order of magnitude, could provide a simplified model in the form of a first order ordinary differential equation, with stochastic forcing provided by the boundary layer skin friction fluctuations. It is important to note that the source of these fluctuations can be either upstream or downstream of separation, and not necessarily the upstream turbulent boundary layer. Remarkably, this analysis results in exactly the same model equation as was originally proposed without proof by Plotkin (1975). The prediction of this type of model is shown with filled symbols on figure 8 , with excellent agreement between the model and both LES and experiment. Recent comparisons with experimental data are included in Poggie et al. (2015). The analysis only requires the flow near the separation to be modelled, suggesting that bubble feedback dynamics (recirculation or acoustic in origin) do not need to be included. It is however, well established that there is a global behaviour of the bubble, usually described as 'breathing' and involving simultaneous changes in bubble length and height, coexisting with forward and backward movements of the shock wave. Recent work by Agostini et al. (2015) describes this behaviour in detail, as well as offering a conceptual model that links shear layer structures to shock-wave motions. 
Another useful way of looking at the problem is provided by the recent work of Sartor et al. (2015), who used a resolvent mode analysis to look at the global response of a transonic bump flow problem (the so-called Delery bump). This linearised analysis considers forcing in the problem formulation and the resulting singular value decomposition provides information about the flow response to forcing and about the forcing that gives the largest gain. Sartor did not find any globally unstable modes and showed how the response problem correctly picked out the dynamics of the shock and shear-layer interactions. The optimal forcing showed both upstream and downstream contributions, whereas in practical applications it would be modified by the disturbance environment. For example, it is now known that the lowfrequency response occurs even in the absence of upstream coherent disturbances (Touber \& Sandham, 2009), or indeed any disturbances at all in the laminar case (Sansica et al., 2014). Our current best explanation is therefore that the low-frequency unsteadiness is the linear response of the separating flow to background noise, with the shock acting as a low-pass filter of disturbances that can come from upstream or downstream. Near the separation there is a high mean streamwise pressure gradient, hence the amplitude of the wall pressure fluctuations at this point is high.

Within the spanwise-homogeneous framework, other configurations are possible, including the combination of ramp-type flows with expansions. Grilli et al. (2012) considered a compression ramp followed by an expansion corner, observing the expected unsteady behaviour near the ramp and analysing this in terms of a dynamic mode decomposition. Fang et al. (2015) considered the opposite combination of an expansion fan followed by a compression corner. In this case turbulent structures are strongly suppressed by the expansion and there is not a full recovery of the flow after the first distortion. Clear Görtler vortices were observed in the separated shear layer associated with the ramp.

\subsection{Sidewall interactions}

In this final section we consider a fully 3D flow that in principle contains many of the phenomena reported in the previous sections. SWBLI interactions with sidewalls links two classes of interactions: a swept SWBLI on the side wall, with the initial shock generator playing the role of a fin embedded in the sidewall boundary layer, and the main oblique SWBLI on a test plate. The problem also includes the possible interaction between the two regions in the corner area which may have its own vortex and separation structure. Eagle \& Driscoll (2014) used stereo PIV to measure all three velocity components, showing details of the corner vortices and showing the need to consider the full 3D problem for shock interactions in low 


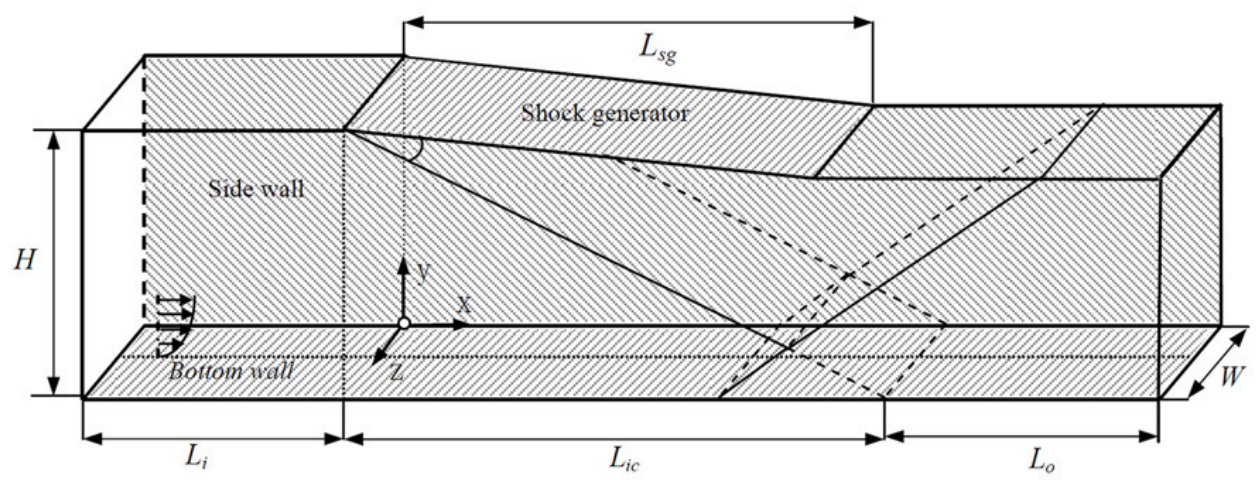

Figure 9: Schematic of SWBLI in a 3D duct with width $W$ and height $H$, from Wang et al. (2015).

aspect ratio ducts. A test geometry suitable for CFD validation was described by Helmer et al. (2012) and Campo \& Eaton (2015), who pointed out a steepening of the main incident shock as it encounters the sidewall boundary layer.

Wall-resolved LES of such configurations is expensive, taking into account the numerical resolution to resolve the SWBLI on the test plate and side walls together with the long run times needed if the low-frequency dynamics are to be investigated. Various alternatives to wall-resolved LES have been tested, including stimulated DES approach of Garnier (2009) mentioned earlier. Garnier used the method to show how the strength of the interaction near the centreline is increased in low aspect-ratio ducts and found the largest pressure fluctuations to be in the corner region. Bermejo-Moreno et al. (2014) used a wall-modelled LES approach based on a sub-grid treatment computing the boundary-layer equations with a mixing-length eddy viscosity. Results were compared with experimental data from Helmer et al. (2012), showing low-frequency oscillations and that the wall-modelled LES are in reasonable quantitative agreement with experimental measurements.

To illustrate the flow phenomena, we consider the configuration from Wang et al. (2015), which included both sidewalls in an $M_{\infty}=2.7$ interaction of an oblique shock with a flat plate boundary layer and was simulated using wall-resolved LES. The $M_{\infty}=2.7$ upstream boundary layer is not expected to experience strong compressibility effects until it enters the region of interaction with an oblique shock that is strong enough to separate the flow. Structures in the outer part of the boundary layer will experience shock/turbulence interaction and the separated shear flow will be affected by compressibility. In addition there are sidewall and corner interactions as well as a strong curvature of the shear layer due to the shock impingement. 


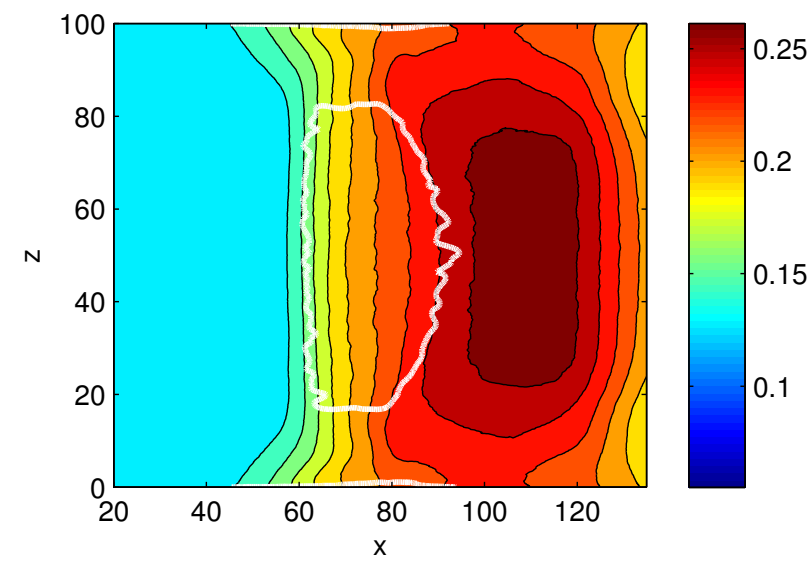

Figure 10: Mean pressure contours on the lower wall showing the pressure rise through the bubble and end-wall effects. The white contours show the regions of reverse flow adjacent to the wall.

Details of the simulations are given in Wang et al. (2015), with the basic set up reproduced in figure 9. After running the simulations all distances have been rescaled to units of mm in the corresponding experiment, whereas other quantities are shown in dimensionless form. The case is set up as a wall-resolved LES and a grid refinement study was run to show the sensitivity of the solution to the grid in each direction separately. The simulations shown here were run on a grid of $480 \times 416 \times 758$ points in the streamwise, normal and spanwise directions respectively. The inflow turbulent boundary is set using the same digital filter approach used by Touber (2010), with the inflow thickness adjusted to give a target Reynolds number of $R e_{\theta}=4300$ based on the boundary layer at the shock impingement location, but in a case with no shock. The interaction is located sufficiently far downstream of the inflow to give the boundary layer enough time to relax to an equilibrium condition, measured by an acceptable comparison of the van Driest scaled profile with incompressible flow data at the same Reynolds number. The cases were run for both sidewalls, since a test simulation with a symmetry plane in the spanwise direction gave unphysical results near that plane due to the constraints imposed of the turbulence by this boundary condition. The upper boundary condition is designed to mimic a shock generator in an experiment. Upstream of the shock generator a characteristic boundary condition was applied. On the shock generator a no-slip condition was set, and then downstream of it the boundary condition was changed back to characteristic. Care was taken to ensure that the expansion wave emanating from the rear of the shock generator impacted on the lower test plate far enough downstream of the interaction not to affect the pressure and skin friction distributions within the interaction. 
The shock generator (with angle $9^{\circ}$ ) generates an oblique shock. Depending on the aspect ratio $W / H$, where $W$ is the width between the two sidewalls and $H$ is the height of the leading edge of the shock generator above the plate, there are different types of interaction. In particular for a case with $W / H=4$ it was shown that there is a region of genuinely spanindependent mean flow near the centreline, with good agreement with a simulation run with spanwise periodic boundary conditions. This is because the sidewall-induced modifications to the impinging shock have not reached the centreline within the interaction region. In contrast, a case with $W / H=1$ was shown to be completely $3 \mathrm{D}$ in the mean, as in Bermejo-Moreno et al. (2014). Here we consider the intermediate case $W / H=2$, for which contours of surface pressure are shown on figure 10. The white contour line marks the edge of the reverse flow region, with the separation line seen to be more two-dimensional than the reattachment line. Clear corner effects are observed, with the wall pressure rising earlier near the corners and with a small corner separation.

The nature of the interaction can be explained by tracing the effects back to the shock generator. The generated shock thickens the sidewall boundary layer. The pressure rise is thus smeared out and the start of the pressure rise precedes the shock location. Another interesting feature is that the shock does not penetrate the sidewall boundary layer down to the sonic line, but only down to a swept sonic condition. Wang et al. (2015) defined a penetration Mach number by $M_{p}=M_{\infty} \cos \beta$, where $\beta$ is the oblique shock angle, and showed that this matched the actual penetration Mach number observed in the simulations. Thus only the very outermost part of the sidewall turbulent boundary layers was exposed to a shock wave. The leading edge of the sidewall interaction reaches the lower wall first (ahead of the main shock induced separation) and reflects in the corner, but causing only a small corner separation. The effect of the interaction with the lower surface was clarified by running a separate simulation with a slip-wall lower boundary condition. This showed that significant spanwise flow is developed irrespective of the lower boundary condition.

In the present contribution we consider some more aspects of the WH2 case from Wang et al. (2015). Figure 11 shows contours on the centreline of the mean streamwise velocity (a), mean pressure (b) and the three normal components of the Reynolds stress (c-e). Also shown on each figure are three white lines corresponding from top to bottom to the sonic line (local $M=1$, solid white line), a streamline located near the centre of the detached shear layer (dashed white line) and a line with zero mean streamwise velocity (solid white line), with reverse flow below this line. The shock pattern is best observed from figure 11b) where the incoming shock crosses the compression waves caused by the boundary layer thickening, 
(a)

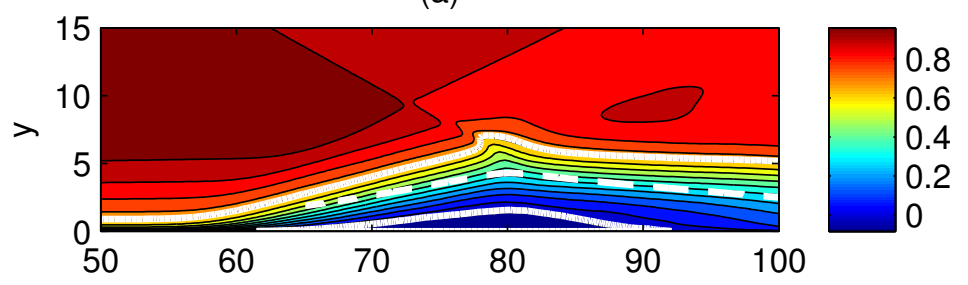

(b)

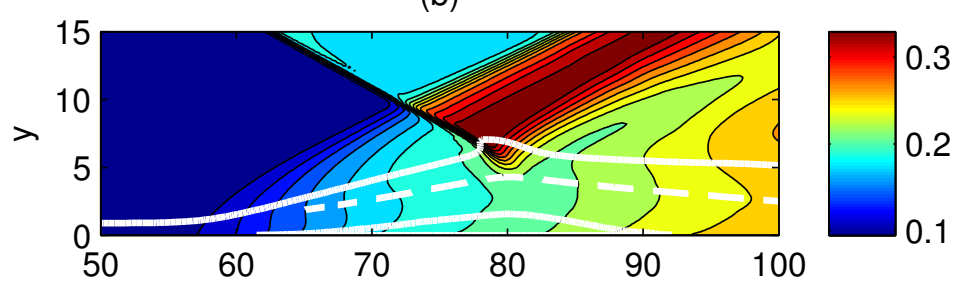

(c)

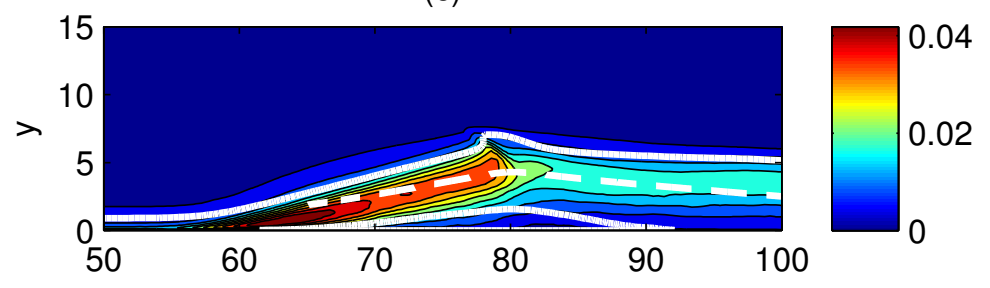

(d)

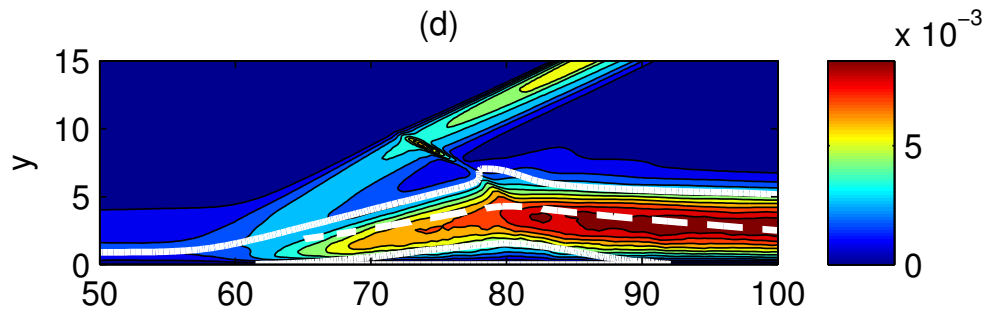

(e)

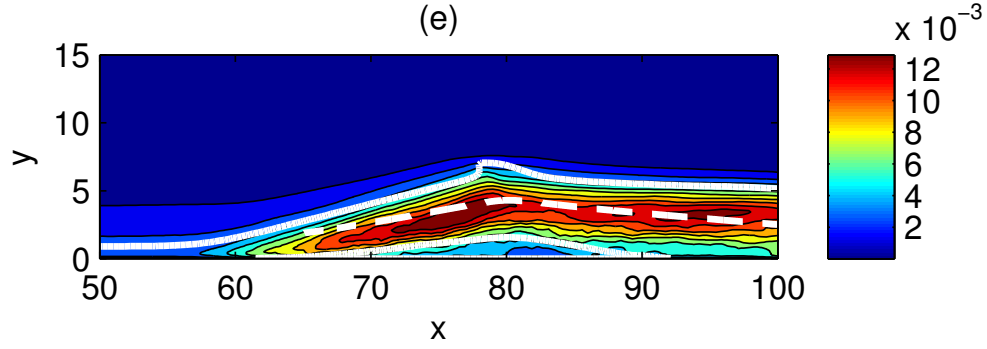

Figure 11: Mean flow and turbulent stresses in the interaction region: (a) velocity, (b) pressure (c) $\overline{u^{\prime 2}}$, (d) $\overline{v^{\prime} 2}$ (e) $\overline{w^{\prime 2}}$. The upper white contour in each frame shows the sonic line and the lower contour shows $\bar{u}=0$. The dashed line is a streamline. 
which starts upstream of the interaction. These reflected waves coalesce into a reflected shock. It can be seen how the incoming shock penetrates approximately to the sonic line and how the incoming shock is reflected as an expansion wave, with the shear-layer streamline then deflected downwards. This pattern is well known from previous studies and is representative of previous LES and experiments.

Figure 11(c-e) should in principle show the effect of turbulence in the outer part of the boundary layer passing through a shock wave that might be compared to previous work on normal-shock/turbulence interaction. However, on closer inspection, that problem seems of little relevance here, since the changes in the turbulence are dominated by the shear layer. In figure $11(\mathrm{c})$ it is just possible to see a small increase in the magnitude of $\overline{u^{\prime 2}}$ in the region above the sonic line. Instead, the streamwise Reynolds stress is dominated by the growth of turbulence in the detached shear layer, reaching a maximum at $x=139$. Interestingly the peak shear stress doesn't exactly align with the streamline in these pictures.

Even more remarkable in figure 11(c) is the strong reduction in the streamwise component of the normal Reynolds stresses after the apex of the bubble. The stress decreases by a factor of approximately two between $x=149$ and $x=155$. Given that the local vorticity thickness is about 6 , this reduction occurs within one vorticity thickness (and substantially less than one boundary layer $99 \%$ thickness). The explanation lies with what might be termed an indirect compressibility effect, based on the shear layer curvature near the apex of the bubble. For the streamline shown in figure 11 the minimum radius of curvature was determined to be 6.9. The direction of the curvature (opposite to the Görtler instability) is stabilizing and such a level of curvature, with the flow deflected by $15^{\circ}$ within one vorticity thickness, is extreme. The curvature arises due to the reflection of the shock wave from the top of the bubble as an expansion, with subsequent flow turning only smeared over one boundary layer thickness. For a turbulence model to cope well with such a shock-induced separation, not only would it need to treat compressibility correctly, but also the stabilising effects of extremely strong curvature. In contrast to the behaviour of $\overline{u^{\prime 2}}$ it can be seen that $\overline{v^{\prime 2}}$ and $\overline{w^{\prime 2}}$ are relatively unaffected by the sudden change of flow direction. Such strong curvature effects may be rather common in shocked flows. For example the measurements of Edgington-Mitchell et al. (2014) show strong reductions in streamwise velocity fluctuations in regions with high turning at shock reflection points in a screeching underexpanded axisymmetric jet.

Figure 12 shows the velocity profile at separation and another two profiles through the separated flow, one upstream and one downstream of the bubble apex. For these two profiles the effective (convective) Mach number was estimated by taking the velocity difference 


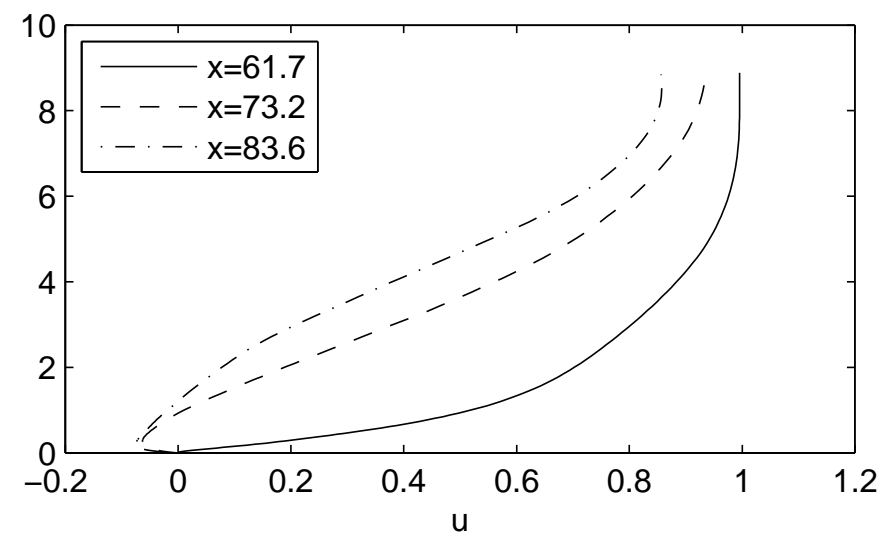

Figure 12: Velocity profiles at separation (solid line) and at two locations within the bubble.

between the external flow (under the shock waves) and the maximum reverse flow and the sound speed at the same two locations. This gave $M_{c}=1.0$ for the upstream profile and $M_{c}=0.9$ for the downstream profile (it may be noted that the freestream is slower at the second location due to the shock waves leading to the lower $M_{c}$ ). Based on the arguments of the previous section, these levels of $M_{c}$ suggest significant compressibility effects on the turbulence. However the profiles are not well approximated by the hyperbolic tangents used in the previous section, and of course a wall is present here, so additional stability calculations are required to check the relevance of the previous results.

An impression of the instantaneous flow can be obtained from figure 13, which in part (a) shows an $x-y$ plane at the duct centreline and in part (b) shows a cut in a $y-z$ plane at $x=73.2$. In figure 13 (b) the sidewall boundary layer is visible and it can be seen that there is a significant penetration of high momentum fluid close to the wall, as also seen by BermejoMoreno et al. (2014). This effect is partly responsible for the mean flow to be attached in a region close to the sidewalls (cf. figure 10) and for the corner separation to be small. The horizontal contour lines present in the freestream are cuts through the impinging and reflected shock waves. In figure 13(a) it can be seen that the detached shear layer develops a wavy motion with a wavelength of perhaps $8-10 \mathrm{~mm}$, while in figure $13(\mathrm{~b})$ it can be seen that there are a series of undulations in the outer part of the boundary layer with a wavelength of approximately $6 \mathrm{~mm}$. The resulting wave angles are around $60^{\circ}$, which is not out of line with the $\theta-M_{c}$ diagram shown on figure 3 , given that the local $M_{c}$ is about one. More comparisons 
(a)

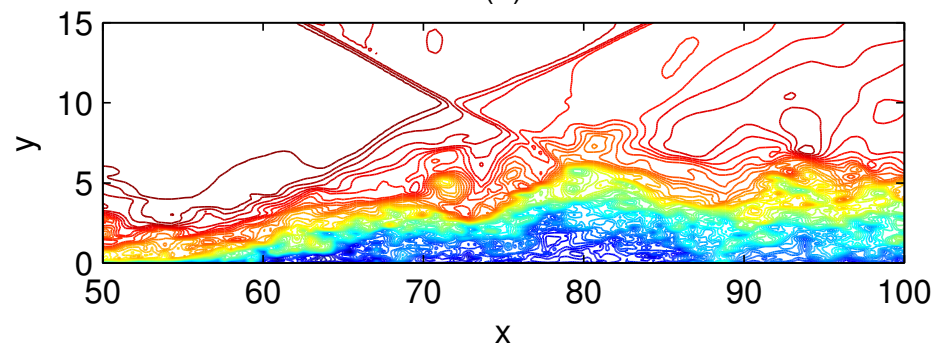

(b)

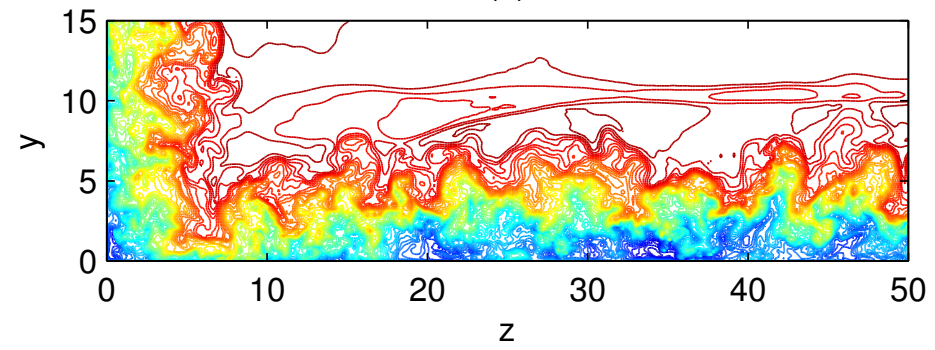

Figure 13: Contours of instantaneous streamwise velocity (a) in an $x-y$ plane at the centreline and (b) in a $y-z$ plane at $x=73.2$.

with stability theory are needed to confirm the potential role of inflectional instability in the generation of these structures. The structures themselves appear to be manifestations of the 'medium' or 'intermediate' frequency band seen in discussed by Aubard et al. (2013) and Agostini et al. (2015).

\section{Conclusions}

An overview of compressible turbulence and SWBLI has been provided, focusing on canonical problems in shock-free and shocked shear flows. An important aspect of the shock-free canonical flows is the fundamental and surprising difference in the effect of Mach number on different flows. Turbulent spots and mixing layers are two model problems where compressibility has a very strong effect, whereas only minor changes are seen in wall-attached ZPG boundary layers. To resolve these differences we have applied a stability map from the mixing layer to other flows in order to better understand the effect of Mach number. The stability map for the mixing layer shows how shear layer instability growth rates reduce with convective Mach number and how the variation of growth rate with wave angle also changes, leading to oblique modes being most unstable and also to a cut-off of modes with supersonic 
relative Mach number. By considering compressibility effects to enter mainly at large scale, this shows how large-scale structures and hence turbulence production can be modified by compressibility. Effective convective Mach numbers have been defined for turbulent spots and for boundary layers under pressure gradients to show how significant compressibility effects may be present in turbulent spots and in equilibrium boundary layers under adverse pressure gradient conditions.

The SWBLI review has focused on the numerical simulation of oblique and ramp SWBLI for which much of the previous research has been carried out, providing more complete and complementary information compared to laboratory experiments, even though the latter are also developing rapidly based on the application of advanced laser-based diagnostics. Spanperiodic simulations have enabled the physics of shock interaction to be explored in detail, including the low-frequency unsteadiness of interactions. The most recent developments have been in the development of simulation capabilities for flows with multiple interactions including oblique SWBLI with sidewalls. In particular, we have considered the development of the mean flow and Reynolds stresses in a 3D SWBLI problem with side wall effects included in the simulation. The high convective Mach numbers within the separation bubble suggest strong compressibility effects on the turbulence. The shock impinges near the apex of the bubble and is reflected as an expansion wave. The associated turning of the flow was shown to have a strong stabilising effect, leading to a reduction in turbulence intensity by over a factor of two. This is an example of an indirect compressibility effect, where the turbulence is governed by streamline curvature, which exists only due to the shock impingement.

\section{Acknowledgements}

The author would like to thank Bo Wang and Satya Jammy for providing databases for the 3D SWBLI case. Data statement: No additional data was generated in this work. Funding: This work would not have been possible wthout the continued support of the UK Turbulence Consortium, under grants EP/G069581/1 and EP/L000261/1. Conflict of interest: The author declares that he has no conflict of interest. A preliminary version of this paper was

presented at the 9th International Symposium on Turbulence and Shear Flow Phenomena (TSFP-9) Conference, June 30th to July 3rd 2015, Melbourne, Australia.

\section{References}

Adams, N.A. 1998 Direct numerical simulation of turbulent compression ramp flow. Theor. Comp. Fluid Dyn. 12 (2), 109-129. 
Adams, N.A. 2000 Direct simulation of the turbulent boundary layer along a compression ramp at $\mathrm{M}=3$ and Re-theta=1685. J. Fluid Mech. 420, 47-83.

Agostini, L., Larcheveque, L. \& Dupont, P. 2015 Mechanism of shock unsteadiness in separated shock/boundary-layer interactions. Phys. Fluids 27 (12).

Aubard, G., Gloerfelt, X. \& Robinet, J. C. 2013 Large-Eddy Simulation of Broadband Unsteadiness in a Shock/Boundary-Layer Interaction. AIAA Journal 51 (10), 2395-2409.

Babinsky, H. \& Harvey, J. 2011 Shock Wave-Boundary-Layer Interactions. Cambridge.

Barre, S. \& Bonnet, J. P. 2015 Detailed experimental study of a highly compressible supersonic turbulent plane mixing layer and comparison with most recent DNS results: "Towards an accurate description of compressibility effects in supersonic free shear flows". Int. J. Heat and Fluid Flow 51, 324-334.

Bermejo-Moreno, I., Campo, L., Larsson, J., Bodart, J., Helmer, D. \& Eaton, J.K. 2014 Confinement effects in shock wave/turbulent boundary layer interactions through wallmodelled large-eddy simulations. J. Fluid Mech. 758, 5-62.

Birch, S. F. \& Eggers, J. M. 1973 A critical review of the experimental data for developed free turbulent shear layers. Tech. Rep. 32. NASA-SP.

Bradshaw, P. 1977 Compressible turbulent shear layers. Ann. Rev. Fluid Mech. 9, 33-54.

Breidenthal, R.E. 1992 Sonic eddy - a model for compressible turbulence. AIAA Journal 30 (1), 101-104.

Brinkerhoff, J.R. \& Yaras, M.I. 2014 Numerical investigation of the generation and growth of coherent flow structures in a triggered turbulent spot. J. Fluid Mech. 759, 257-294.

Brown, G.L. \& Roshko, A. 1974 Density effects and large structure in turbulent mixing layers. J. Fluid Mech. 64, 775-816.

Campo, L.M. \& Eaton, J.K. 2015 Shock boundary layer interactions in a low aspect ratio duct. Int. J. Heat Fluid Flow 51, 353-371.

Casper, K.M., Beresh, S.J. \& Schneider, S.P. 2014 Pressure fluctuations beneath instability wavepackets and turbulent spots in a hypersonic boundary layer. J. Fluid Mech. 756, 10581091.

Chu, B.-T. \& Kovásznay, S. G. 1958 Non-linear interactions in a viscous heat-conducting compressible gas. Journal of Fluid Mechanics 3, 515-522.

Clemens, N.T. \& Narayanaswamy, V. 2014 Low frequency unsteadiness of shock wave/turbulent boundary layer interactions. Ann. Rev. Fluid Mech. 46, 469-492.

Coleman, G.N., Kim, J. \& Moser, R.D. 1995 A numerical study of turbulent supersonic isothermal-wall channel flow. J. Fluid Mech. 305, 159-183. 
Doerffer, P., Hirsch, C., Dussauge, J.-P., Babinsky, H. \& Barakos, G.N. 2010 Unsteady Effect of Shock Wave Induced Separation, Notes on Numerical Fluid Mechanics and Multidisciplinary Design, vol. 114. Springer.

Dolling, D.S. 2001 Fifty years of shock-wave/boundary-layer interaction research: What next? AIAA Journal 39 (8), 1517-1531.

Duan, L. \& Martin, M. P. 2011 Direct numerical simulation of hypersonic turbulent boundary layers. Part 4. Effect of high enthalpy. J. Fluid Mech. 684, 25-59.

Ducros, F., Ferrand, V., Nicoud, F., Weber, C., Darracq, D., Gacherieu, C. \& Poinsot, T. 1999 Large-eddy simulation of the shock turbulence interaction. J. Comp. Phys. 152 (2), $517-549$.

Dupont, P., Haddad, C. \& Debiève, J.F. 2006 Space and time organization in a shock-induced separated boundary layer. J. Fluid Mech. 559, 255-277.

Eagle, W.E. \& Driscoll, J.F. 2014 Shock wave-boundary layer interactions in rectangular inlets: three-dimensional separation topology and critical points. J. Fluid Mech. 756, 328353.

Edgington-Mitchell, D., Oberleithner, K., Honnery, D.R. \& Soria, J. 2014 Coherent structure and sound production in the helical mode of a screeching axisymmetric jet. J. Fluid Mech. $\mathbf{7 4 8}, 822-847$.

Fang, J., Yao, Y., Zheltovodov, A.A., Li, Z. \& Lu, L. 2015 Direct numerical simulation of supersonic turbulent flows around a tandem expansion-compression corner. Phys. Fluids $27(12)$.

Fischer, M.C. 1972 Spreading of a turbulent disturbance. AIAA Journal 10, 957-959.

Gad-El-Hak, M., Blackwelder, R.F. \& Riley, J.J. 1981 On the growth of turbulent regions in laminar boundary-layers. J. Fluid Mech. 110, 73-95.

Gaitonde, D.V. 2015 Progress in shock wave/boundary layer interactions. Prog. Aero. Sci. 72 (SI), 80-99.

Ganapathisubramani, B., Clemens, N.T. \& Dolling, D.S. 2007 Effects of upstream boundary layer on the unsteadiness of shock-induced separation. J. Fluid Mech. 585, 369-394.

Garnier, E. 2009 Stimulated detached eddy simulation of three-dimensional shock/boundary layer interaction. Shock Waves 19 (6), 479-486.

Garnier, E., Adams, N.A. \& Sagaut, P. 2009 Large Eddy Simulation for Compressible Flows. Springer.

Garnier, E., Sagaut, P. \& Deville, M. 2002 Large eddy simulation of shock/boundary-layer interaction. AIAA Journal 40 (10), 1935-1944. 
Gaster, M., Kit, E. \& Wygnanski, I. 1985 Large-scale structures in a forced turbulent mixing layer. J. Fluid Mech. 150, 23-39.

Gatski, T.B. \& Bonnet, J.P. 2009 Compressibility, Turbulence and High-Speed Flow. Academic Press.

Gerritsen, M. \& Olsson, P. 1996 Designing an efficient solution strategy for fluid flows .1. A stable high order finite difference scheme and sharp shock resolution for the Euler equations. J. Comp. Phys 129 (2), 245-262.

Giepman, R.H.M., Schrijer, F.F.J. \& van Oudheusden, B.W. 2015 High-resolution PIV measurements of a transitional shock wave-boundary layer interaction. Exp. Fluids 56 (6), $1-20$.

Goldstein, D., Chu, J. \& Brown, G. 2015 Lateral spreading mechanism of a turbulent spot an a turbulent wedge. In Proceedings of the International Symposium on Turbulence and Shear Flow Phenomena, pp. Paper 6B-2, 1-6. Melbourne, Australia.

Grilli, M., Schmid, P.J., Hickel, S. \& Adams, N.A. 2012 Analysis of unsteady behaviour in shockwave turbulent boundary layer interaction. J. Fluid Mech. 700, 16-28.

Helmer, D. B., Campo, L. M. \& Eaton, J. K. 2012 Three-dimensional features of a Mach 2.1 shock/boundary layer interaction. Expt. Fluids $\mathbf{5 3}$ (5), 1347-1368.

Hickel, S., Egerer, C.P. \& Larsson, J. 2014 Subgrid-scale modeling for implicit large eddy simulation of compressible flows and shock-turbulence interaction. Phys. Fluids 26 (10).

Huang, P.G., Coleman, G.N. \& Bradshaw, P. 1995 Compressible turbulent channel flows: DNS results and modelling. J. Fluid Mech. 305, 185-218.

Jocksch, A. \& Kleiser, L. 2008 Growth of turbulent spots in high-speed boundary layers on a flat plate. Int. J. Heat and Fluid Flow 29 (6), 1543-1557.

Johnsen, E., Larsson, J., Bhagatwala, A.V., Cabot, W.H., Moin, P., Olson, B.J., Rawat, P.S., Shankar, S.K., Sjoegreen, B., Yee, H.C., Zhong, X. \& Lele, S.K. 2010 Assessment of highresolution methods for numerical simulations of compressible turbulence with shock waves. J. Comp. Phys. 229 (4), 1213-1237.

Krishnan, L. \& Sandham, N. D. 2006 Effect of Mach number on the structure of turbulent spots. J. Fluid Mech. 566, 225-234.

Kumar, G., Bertsch, Rebecca L. \& Girimaji, Sharath S. 2014 Stabilizing action of pressure in homogeneous compressible shear flows: effect of Mach number and perturbation obliqueness. J. Fluid Mech. 760, 540-566.

Larsson, J., Bermejo-Moreno, I. \& Lele, S. K. 2013 Reynolds- and Mach-number effects in canonical shock-turbulence interaction. J. Fluid Mech. 717, 293-321.

Lawal, A.A. 2002 Direct numerical simulation of transonic shock/boundary-layer interactions. $\mathrm{PhD}$ thesis, University of Southampton, Southampton, U.K. 
Maeder, T., Adams, N.A. \& Kleiser, L.. 2001 Direct simulation of turbulent supersonic boundary layers by an extended temporal approach. J. Fluid Mech. 429, 187-216.

Mahesh, K., Lele, S.K. \& Moin, P. 1997 The influence of entropy fluctuations on the interaction of turbulence with a shock wave. J. Fluid Mech. 334, 353-379.

Matheis, J. \& Hickel, S. 2015 On the transition between regular and irregular shock patterns of shock-wave/boundary-layer interactions. J. Fluid Mech. 776, 200-234.

Mayer, C.S.J., von Terzi, D.A. \& Fasel, H.F. 2011 Direct numerical simulation of complete transition to turbulence via oblique breakdown at Mach 3. J. Fluid Mech. 674, 5-42.

Monkewitz, P.A. \& Huerre, P. 1982 Influence of the velocity ratio on the spatial instability of mixing layers. Phys. Fluids 25 (7), 1137-1143.

Morris, P.J., Giridharan, M.G. \& Lilley, G.M. 1990 On the turbulent mixing of compressible free shear layers. Proc. Roy. Soc.-Mathematical and Phys. Sciences 431 (1882), 219-243.

Pagella, A., Babucke, A. \& Rist, U. 2004 Two-dimensional numerical investigations of smallamplitude disturbances in a boundary layer at $\mathrm{Ma}=4.8$ : Compression corner versus impinging shock wave. Phys. Fluids 16 (7), 2272-2281.

Pantano, C. \& Sarkar, S. 2002 A study of compressibility effects in the high-speed turbulent shear layer using direct simulation. J. Fluid Mech. 451, 329-371.

Papamoschou, D. \& Roshko, A. 1988 The compressible turbulent shear-layer - an experimental-study. J. Fluid Mech. 197, 453-477.

Piponniau, S., Dussauge, J.P., Debiève, J.F. \& Dupont, P. 2009 A simple model for lowfrequency unsteadiness in shock-induced separation. J. Fluid Mech. 629, 87-108.

Pirozzoli, S. 2011 Numerical Methods for High-Speed Flows. In Ann. Rev. Fluid Mech. (ed. Davis, S.H. and Moin, P.), Annual Review of Fluid Mechanics, vol. 43, pp. 163-194. Annual Reviews Inc.

Pirozzoli, S., Bernardini, M. \& Grasso, F. 2010 Direct numerical simulation of transonic shock/boundary layer interaction under conditions of incipient separation. J. Fluid Mech. 657, 361-393.

Pirozzoli, S. \& Grasso, F. 2006 Direct numerical simulation of impinging shock wave/turbulent boundary layer interaction at $\mathrm{M}=2.25$. Phys. Fluids 18 (6).

Pirozzoli, S., Grasso, F. \& Gatski, T.B. 2004 Direct numerical simulation and analysis of a spatially evolving supersonic turbulent boundary layer at $\mathrm{M}=2.25$. Phys. Fluids 16 (3), $530-545$.

Plotkin, K.J. 1975 Shock-wave oscillation driven by turbulent boundary-layer fluctuations. AIAA Journal 13 (8), 1036-1040. 
Poggie, J., Bisek, N.J., Kimmel, R.L. \& Stanfield, S.A. 2015 Spectral Characteristics of Separation Shock Unsteadiness. AIAA Journal 53 (1), 200-214.

Ragab, S.A. \& Wu, J.L. 1989 Linear instabilities in two-dimensional compressible mixing layers. Physics of Fluids A-Fluid Dynamics 1 (6), 957-966.

Redford, J. A., Sandham, N. D. \& Roberts, G. T. 2012 Numerical simulations of turbulent spots in supersonic boundary layers: Effects of Mach number and wall temperature. Progress in Aerospace Sciences 52 (SI), 67-79.

Robinet, J.-Ch. 2007 Bifurcations in shock-wave/laminar-boundary-layer interaction: global instability approach. J. Fluid Mech. 579, 85-112.

Ryu, J. \& Livescu, D. 2014 Turbulence structure behind the shock in canonical shock-vortical turbulence interaction. J. Fluid Mech. $\mathbf{7 5 6 .}$

Sandham, N.D., Li, Q. \& Yee, H.C. 2002 Entropy splitting for high-order numerical simulation of compressible turbulence. J. Comp. Phys. 178 (2), 307-322.

Sandham, N.D. \& Reynolds, W.C. 1990 Compressible mixing layer - linear theory and direct simulation. AIAA Journal 28 (4), 618-624.

Sandham, N.D. \& Reynolds, W.C. 1991 Three-dimensional simulations of large eddies in the compressible mixing layer. J. Fluid Mech. 224, 133-158.

Sandham, N.D. \& Sandberg, R.D. 2009 Direct numerical simulation of the early development of a turbulent mixing layer downstream of a splitter plate. Journal of Turbulence 10 (1), $1-17$.

Sansica, A., Sandham, N.D. \& Hu, Z. 2014 Forced response of a laminar shock-induced separation bubble. Phy. Fluids 26, 957-959.

Sarkar, S. 1995 The stabilizing effect of compressibility in turbulent shear-flow. J. Fluid Mech. 282, $163-186$.

Sarkar, S., Erlebacher, G., Hussaini, M.Y. \& Kreiss, H.O. 1991 The analysis and modeling of dilatational terms in compressible turbulence. J. Fluid Mech. 227, 473-493.

Sartor, F., Mettot, C., Bur, R. \& Sipp, D. 2015 Unsteadiness in transonic shockwave/boundary-layer interactions: experimental investigation and global stability analysis. J. Fluid Mech. 781, 550-577.

Slessor, M.D., Bond, C.L. \& Dimotakis, P.E. 1998 Turbulent shear-layer mixing at high reynolds numbers: effects of inflow conditions. J. Fluid Mech. 376, 115-138.

Smits, A.J. \& Dussauge, J.-P. 2006 Turbulent Shear Layers in Supersonic Flow, 3rd edn. Springer.

Souverein, L.J., Dupont, P., Debieve, J.-F., Dussauge, J.-P., van Oudheusden, B.W. \& Scarano, F. 2010 Effect of Interaction Strength on Unsteadiness in Turbulent Shock-WaveInduced Separations. AIAA Journal 48 (7), 1480-1493. 
Suzuki, T. \& Colonius, T. 2006 Instability waves in a subsonic round jet detected using a near-field phased microphone array. J. Fluid Mech. 565, 197-226.

Touber, E. 2010 Unsteadiness in shock-wave/boundary-layer interactions. PhD thesis, University of Southampton, Southampton, U.K.

Touber, E. \& Sandham, N.D. 2009 Large-eddy simulation of low-frequency unsteadiness in a turbulent shock-induced separation bubble. Theo. and Comp. Fluid Dyn. 23 (2), 79-107.

Touber, E. \& Sandham, N. D. 2011 Low-order stochastic modelling of low-frequency motions in reflected shock-wave/boundary-layer interactions. J. Fluid Mech. 671, 417-465.

Vreman, A.W., Sandham, N.D. \& Luo, K.H. 1996 Compressible mixing layer growth rate and turbulence characteristics. J. Fluid Mech. 320, 235-258.

Vreman, B. 1995 Direct and large-eddy simulation of the compressible mixing layer. PhD thesis, University of Twente, Enschede, The Netherlands.

Wang, B., Sandham, N.D., Hu, Z. \& Liu, W. 2015 Numerical study of oblique shockwave/boundary-layer interaction considering sidewall effects. J. Fluid Mech. 767, 526-561.

Wilcox, D.C. 2006 Turbulence Modeling for CFD, 3rd edn. McGraw-Hill.

Yee, H.C., Sandham, N.D. \& Djomehri, M.J. 1999 Low-dissipative high-order shock-capturing methods using characteristic-based filters. J. Comp. Phys. 150 (1), 199-238.

Yoder, D. A., DeBonis, J. R. \& Georgiadis, N. J. 2015 Modeling of turbulent free shear flows. Comp. Fluids 117, 212-232. 